\title{
A New Decomposition Method for Attractor Detection in Large Synchronous Boolean Networks
}

\author{
Andrzej Mizera ${ }^{1}$, Jun Pang ${ }^{1,2}$, Hongyang $\mathrm{Qu}^{3}$, and Qixia Yuan ${ }^{1 \star}$ \\ 1 Faculty of Science, Technology and Communication, University of Luxembourg \\ ${ }^{2}$ Interdisciplinary Centre for Security, Reliability and Trust, University of Luxembourg \\ 3 Department of Automatic Control \& Systems Engineering, University of Sheffield \\ firstname.lastnameduni.lu, h.qu@sheffield.ac.uk
}

\begin{abstract}
Boolean networks is a well-established formalism for modelling biological systems. An important challenge for analysing a Boolean network is to identify all its attractors. This becomes challenging for large Boolean networks due to the well-known state-space explosion problem. In this paper, we propose a new SCC-based decomposition method for attractor detection in large synchronous Boolean networks. Experimental results show that our proposed method is significantly better in terms of performance when compared to existing methods in the literature.
\end{abstract}

\section{Introduction}

Boolean networks (BN) is a well-established framework widely used for modelling biological systems, such as gene regulatory networks (GRNs). Although it is simple by mainly focusing on the wiring of a system, BN can still capture the important dynamic property of the modelled system, e.g., the attractors. In the literature, attractors are hypothesised to characterise cellular phenotypes [1] or to correspond to functional cellular states such as proliferation, apoptosis, or differentiation [2]. Hence, attractor identification is of vital importance to the study of biological systems modelled as BNs.

Attractors are defined based on the BN's state space (often represented as a transition system or graph), the size of which is exponential in the number of nodes in the network. Therefore, attractor detection becomes non-trivial when it comes to a large network. In the BN framework, algorithms for detecting attractors have been extensively studied in the literature. The first study dates back to the early 2000 s when Somogyi and Greller proposed an enumeration and simulation method [3]. The idea is to enumerate all the possible states and to run simulation from each of them until an attractor is found. This method is largely limited by the network size as its running time grows exponentially with the number of nodes in the BN. Later on, the performance of attractor detection has been greatly improved with the use of two techniques. The first technique is binary decision diagrams (BDDs). This type of methods [4,5] encodes Boolean functions of BNs with BDDs and represents the network's corresponding transition system with BDD structures. Using the BDD operations, the forward and backward reachable states can be often efficiently computed. Detecting attractors is then

\footnotetext{
^ Supported by the National Research Fund, Luxembourg (grant 7814267).
} 
reduced to finding fix point sets of states in the corresponding transition system. The other technique makes use of satisfiability (SAT) solvers. It transforms attractor detection in BNs into a SAT problem [6]. An unfolding of the transition relation of the BN for a bounded number of steps is represented as a propositional formula. The formula is then solved by a SAT solver to identify a valid path in the state transition system of the BN. The process is repeated iteratively for larger and larger bounded numbers of steps until all attractors are identified. The efficiency of the algorithm largely relies on the number of unfolding steps required and the number of nodes in the BN. Recently, a few decomposition methods [7-9] were proposed to deal with large BNs. The main idea is to decompose a large BN into small components based on its structure, to detect attractors of the small components, and then recover the attractors of the original BN.

In this paper, we propose a new decomposition method for attractor detection in BNs, in particular, in large synchronous BNs, where all the nodes are updated synchronously at each time point. Considering the fact that a few decomposition methods have already been introduced, we explain our new method by showing its main differences from the existing ones. First, our method carefully considers the semantics of synchronous BNs and thus it does not encounter a problem that the method proposed in [7] does. We explain this in more details in Section 3. Second, our new method considers the dependency relation among different sub-networks when detecting attractors of them while our previous method [8] does not require this. We show with experimental results that this consideration can significantly improve the performance of attractor detection in large BNs. Further, the decomposition method in [9] is designed for asynchronous networks while here we extend it for synchronous networks. As a consequence, the key operation realisation for the synchronous BNs is completely re-designed with respect to the one for asynchronous BNs in [9].

\section{Preliminaries}

\subsection{Boolean networks}

A Boolean network (BN) is composed of two elements: binary-valued nodes, which represents elements of a biological system, and Boolean functions, which represent interactions between the elements. The concept of BNs was first introduced in 1969 by S. Kauffman for analysing the dynamical properties of GRNs [10], where each gene was assumed to be in only one of two possible states: ON/OFF.

Definition 1 (Boolean network). A Boolean network $G(V, f)$ consists of a set of nodes $V=\left\{v_{1}, v_{2}, \ldots, v_{n}\right\}$, also referred to as genes, and a vector of Boolean functions $\boldsymbol{f}=\left(f_{1}, f_{2}, \ldots, f_{n}\right)$, where $f_{i}$ is a predictor function associated with node $v_{i}(i=$ $1,2, \ldots, n)$. A state of the network is given by a vector $\boldsymbol{x}=\left(x_{1}, x_{2}, \ldots, x_{n}\right) \in\{0,1\}^{n}$, where $x_{i} \in\{0,1\}$ is a value assigned to node $v_{i}$.

Since the nodes are binary, the state space of a BN is exponential in the number of nodes. Each node $v_{i} \in V$ has an associated subset of nodes $\left\{v_{i_{1}}, v_{i_{2}}, \ldots, v_{i_{k(i)}}\right\}$, referred to as the set of parent nodes of $v_{i}$, where $k(i)$ is the number of parent nodes and $1 \leq i_{1}<i_{2}<\cdots<i_{k(i)} \leq n$. Starting from an initial state, the $\mathrm{BN}$ evolves in time 
by transiting from one state to another. The state of the network at a discrete time point $t(t=0,1,2, \ldots)$ is given by a vector $\boldsymbol{x}(t)=\left(x_{1}(t), x_{2}(t), \ldots, x_{n}(t)\right)$, where $x_{i}(t)$ is a binary-valued variable that determines the value of node $v_{i}$ at time point $t$. The value of node $v_{i}$ at time point $t+1$ is given by the predictor function $f_{i}$ applied to the values of the parent nodes of $v_{i}$ at time $t$, i.e., $x_{i}(t+1)=f_{i}\left(x_{i_{1}}(t), x_{i_{2}}(t), \ldots, x_{i_{k(i)}}(t)\right)$. For simplicity, with slight abuse of notation, we use $f_{i}\left(x_{i_{1}}, x_{i_{2}}, \ldots, x_{i_{k(i)}}\right)$ to denote the value of node $v_{i}$ at the next time step. For any $j \in[1, k(i)]$, node $v_{i_{j}}$ is called a parent node of $v_{i}$ and $v_{i}$ is called a child node of $v_{i_{j}}$.

In general, the Boolean predictor functions can be formed by combinations of any logical operators, e.g., logical AND $\wedge$, OR $\vee$, and NEGATION $\neg$, applied to variables associated with the respective parent nodes. The BNs are divided into two types based on the time evolution of their states, i.e., synchronous and asynchronous. In synchronous BNs, values of all the variables are updated simultaneously; while in asynchronous $\mathrm{BNs}$, one variable at a time is randomly selected for update.

In this paper, we focus on synchronous BNs. The transition relation of a synchronous $\mathrm{BN}$ is given by

$$
T(\boldsymbol{x}(t), \boldsymbol{x}(t+1))=\bigwedge_{i=1}^{n}\left(x_{i}(t+1) \leftrightarrow f_{i}\left(x_{i_{1}}(t), x_{i_{2}}(t), \cdots, x_{i_{k_{i}}}(t)\right)\right) .
$$

It states that in every step, all the nodes are updated synchronously according to their Boolean functions.

In many cases, a BN $G(V, \boldsymbol{f})$ is studied as a state transition system. Formally, the definition of state transition system is given as follows.

Definition 2 (State transition system). A state transition system $\mathcal{T}$ is a 3-tuple $\left\langle S, S_{0}\right.$, $T\rangle$ where $S$ is a finite set of states, $S_{0} \subseteq S$ is the initial set of states and $T \subseteq S \times S$ is the transition relation. When $S=S_{0}$, we write $\langle S, T\rangle$.

A BN can be easily modelled as a state transition system: the set $S$ is just the state space of the BN, so there are $2^{n}$ states for a BN with $n$ nodes; the initial set of states $S_{0}$ is the same as $S$; finally, the transition relation $T$ is given by Equation 1 .

Example 1. A BN with 3 nodes is shown in Figure 1a. Its Boolean functions are given as: $f_{1}=\neg\left(x_{1} \wedge x_{2}\right), f_{2}=x_{1} \wedge \neg x_{2}$, and $f_{3}=\neg x_{2}$. In Figure 1a, the three circles $v_{1}, v_{2}$ and $v_{3}$ represent the three nodes of the $\mathrm{BN}$. The edges between nodes represent the interactions between nodes. Applying the transition relation to each of the states, we can get the corresponding state transition system. For better understanding, we demonstrate the state transition system as a state transition graph in this paper. The corresponding state transition graph of this example is shown in Figure 1b.

In the transition graph of Figure $1 \mathrm{~b}$, the three states $(000),(1 * 1)^{4}$ can be reached from each other but no other state can be reached from any of them. This forms an attractor of the BN. The formal definition of an attractor is given as follows.

\footnotetext{
${ }^{4}$ We use $*$ to denote that the bit can have value either 1 or 0 , so $(1 * 1)$ actually denotes two states: 101 and 111 .
} 


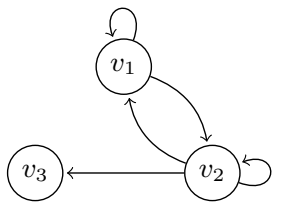

(a) A BN with 3 nodes.

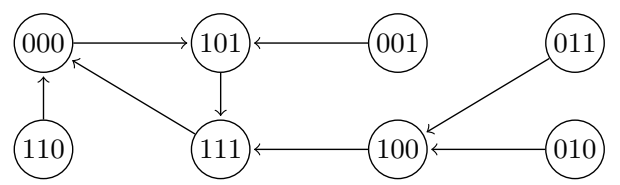

(b) Transition graph of the $\mathrm{BN}$ in Example 1.

Fig. 1: The Boolean network in Example 1 and its state transition graph.

Definition 3 (Attractor of a BN). An attractor of a BN is a set of states satisfying that any state in this set can be reached from any other state in this set and no state in this set can reach any other state that is not in this set.

When analysing an attractor, we often need to identify transition relations between the attractor states. We call an attractor together with its state transition relation an attractor system (AS). The states constituting an attractor are called attractor states. The attractors of a BN characterise its long-run behaviour [11] and are of particular interest due to their biological interpretation.

For synchronous BNs, each state of the network can only have at most one outgoing transition. Therefore, the transition graph of an attractor in a synchronous BN is simply a loop. By detecting all the loops in a synchronous BN, one can identify all its attractors.

Example 2. The BN given in Example 1 has one attractor, i.e., $\{(000),(1 * 1)\}$.

\subsection{Encoding BNs in BDDs}

Binary decision diagrams (BDDs) were introduced by Lee in [12] and Akers in [13] to represent Boolean functions $[12,13]$. BDDs have the advantage of memory efficiency and have been applied in many model checking algorithms to alleviate the state space explosion problem. A BN $G(V, \boldsymbol{f})$ can be modelled as a state transition system, which can then be encoded in a BDD.

Each variable in $V$ can be represented by a binary BDD variable. By slight abuse of notation, we also use $V$ to denote the set of BDD variables. In order to encode the transition relation, another set $V^{\prime}$ of BDD variables, which is a copy of $V$, is introduced: $V$ encodes the possible current states, i.e., $\boldsymbol{x}(t)$, and $V^{\prime}$ encodes the possible next states, i.e., $\boldsymbol{x}(t+1)$. Hence, the transition relation can be viewed as a Boolean function $T: 2^{|V|+\left|V^{\prime}\right|} \rightarrow\{0,1\}$, where values 1 and 0 indicate a valid and an invalid transition, respectively. Our attractor detection algorithm, which will be discussed in the next section, also utilizes two basis functions: $\operatorname{Image}(X, T)=\left\{s^{\prime} \in S \mid \exists s \in\right.$ $X$ such that $\left.\left(s, s^{\prime}\right) \in T\right\}$, which returns the set of target states that can be reached from any state in $X \subseteq S$ with a single transition in $T ; \operatorname{Preimage}(X, T)=\left\{s^{\prime} \in\right.$ $S \mid \exists s \in X$ such that $\left.\left(s^{\prime}, s\right) \in T\right\}$, which returns the set of predecessor states that can reach a state in $X$ with a single transition. To simplify the presentation, we define $\operatorname{Preimage}^{i}(X, T)=\underbrace{\operatorname{Preimage}(\ldots(\operatorname{Preimage}(X, T)))}$ with $\operatorname{Preimage}^{0}(X, T)=X$. 
In this way, the set of all states that can reach a state in $X$ via transitions in $T$ is defined as an iterative procedure Predecessors $(X, T)=\bigcup_{i=0}^{n} \operatorname{Preimage}^{n}(X, T)$ such that Preimage $^{n}(X, T)=$ Preimage $^{n+1}(X, T)$. Given a set of states $X \subseteq S$, the projection $\left.T\right|_{X}$ of $T$ on $X$ is defined as $\left.T\right|_{X}=\left\{\left(s, s^{\prime}\right) \in T \mid s \in X \wedge s^{\prime} \in X\right\}$.

\section{The New Method}

In this section, we describe in details the new SCC-based decomposition method for detecting attractors of large synchronous BNs and we prove its correctness. The method consists of three steps. First, we divide a BN into sub-networks called blocks and this step is performed on the network structure, instead of the state transition system of the network. Second, we detect attractors in blocks. Last, we recover attractors of the original $\mathrm{BN}$ based on attractors of the blocks.

\subsection{Decompose a BN}

We start by giving the formal definition of a block.

Definition 4 (Block). Given a $B N G(V, \boldsymbol{f})$ with $V=\left\{v_{1}, v_{2}, \ldots, v_{n}\right\}$ and $\boldsymbol{f}=\left\{f_{1}\right.$, $\left.f_{2}, \ldots, f_{n}\right\}$, a block $B\left(V^{B}, \boldsymbol{f}^{\boldsymbol{B}}\right)$ is a subset of the network, where $V^{B} \subseteq V$. For any node $v_{i} \in V^{B}$, if $B$ contains all the parent nodes of $v_{i}$, its Boolean function in $B$ remains the same as in $G$, i.e., $f_{i}$; otherwise, the Boolean function is undetermined, meaning that additional information is required to determine the value of $v_{i}$ in $B$. We call the nodes with undetermined Boolean functions as undetermined nodes. We refer to a block as an elementary block if it contains no undetermined nodes.

We consider synchronous networks in this paper and therefore a block is also under the synchronous updating scheme, i.e., all the nodes in the block will be updated at each given time point no matter this node is undetermined or not.

We now introduce a method to construct blocks, using SCC-based decomposition. Formally, the standard graph-theoretical definition of an SCC is as follows.

Definition 5 (SCC). Let $\mathcal{G}$ be a directed graph and $\mathcal{V}$ be its vertices. A strongly connected component (SCC) of $\mathcal{G}$ is a maximal set of vertices $C \subseteq \mathcal{V}$ such that for every pair of vertices $u$ and $v$, there is a directed path from $u$ to $v$ and vice versa.

We first decompose a given BN into SCCs. Figure 2a shows the decomposition of a BN into four SCCs: $\Sigma_{1}, \Sigma_{2}, \Sigma_{3}$, and $\Sigma_{4}$. A node outside an SCC that is a parent to a node in the SCC is referred to as a control node of this SCC. In Figure $2 \mathrm{a}$, node $v_{1}$ is a control node of $\Sigma_{2}$ and $\Sigma_{4}$; node $v_{2}$ is a control node of $\Sigma_{3}$; and node $v_{6}$ is a control node of $\Sigma_{4}$. The SCC $\Sigma_{1}$ does not have any control node. An SCC together with its control nodes forms a block. For example, in Figure $2 \mathrm{a}, \Sigma_{2}$ and its control node $v_{1}$ form one block $B_{2} . \Sigma_{1}$ itself is a block, denoted as $B_{1}$, since the SCC it contains does not have any control node. If a control node in a block $B_{i}$ is a determined node in another block $B_{j}$, block $B_{j}$ is called a parent of block $B_{i}$ and $B_{i}$ is a child of $B_{j}$. By adding directed 


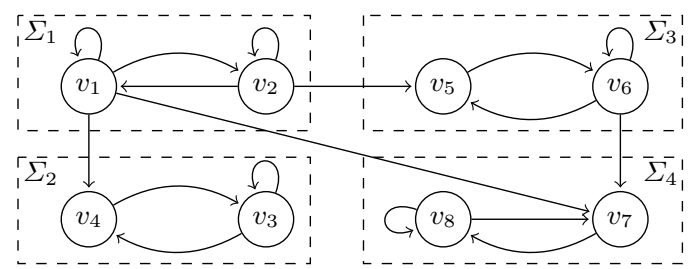

(a) SCC decomposition.

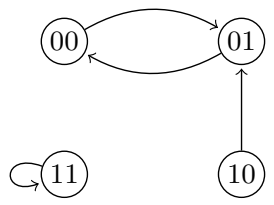

(b) Transition graph of block $B_{1}$.

Fig. 2: SCC decomposition and the transition graph of block $B_{1}$.

edges from all parent blocks to all their child blocks, we form a directed acyclic graph (DAG) of the blocks as the blocks are formed from SCCs. As long as the block graph is guaranteed to be a DAG, other strategies to form blocks can be used. Two blocks can be merged into one larger block. For example, blocks $B_{1}$ and $B_{2}$ can be merged together to form a larger block $B_{1,2}$.

A state of a block is a binary vector of length equal to the size of the block which determines the values of all the nodes in the block. In this paper, we use a number of operations on the states of a BN and its blocks. Their definitions are given below.

Definition 6 (Projection map, Compressed state, Mirror states). For a BN G and its block $B$, where the set of nodes in $B$ is $V^{B}=\left\{v_{1}, v_{2}, \ldots, v_{m}\right\}$ and the set of nodes in $G$ is $V=\left\{v_{1}, v_{2}, \ldots, v_{m}, v_{m+1}, \ldots, v_{n}\right\}$, the projection map $\pi_{B}: X \rightarrow X^{B}$ is given by $\boldsymbol{x}=\left(x_{1}, x_{2}, \ldots, x_{m}, x_{m+1}, \ldots, x_{n}\right) \mapsto \pi_{B}(\boldsymbol{x})=\left(x_{1}, x_{2}, \ldots, x_{m}\right)$. For any set of states $S \subseteq X$, we define $\pi_{B}(S)=\left\{\pi_{B}(\boldsymbol{x}): \boldsymbol{x} \in S\right\}$. The projected state $\pi_{B}(\boldsymbol{x})$ is called a compressed state of $\boldsymbol{x}$. For any state $\boldsymbol{x}^{B} \in X^{B}$, we define its set of mirror states in $G$ as $\mathcal{M}_{G}\left(\boldsymbol{x}^{B}\right)=\left\{\boldsymbol{x} \mid \pi_{B}(\boldsymbol{x})=\boldsymbol{x}^{B}\right\}$. For any set of states $S^{B} \subseteq X^{B}$, its set of mirror states is $\mathcal{M}_{G}\left(S^{B}\right)=\left\{\boldsymbol{x} \mid \pi_{B}(\boldsymbol{x}) \in S^{B}\right\}$.

The concept of projection map can be naturally extended to blocks. Given a block with nodes $V^{B}=\left\{v_{1}, v_{2}, \ldots, v_{m}\right\}$, let $V^{B^{\prime}}=\left\{v_{1}, v_{2}, \ldots, v_{j}\right\} \subseteq V^{B}$. We can define $\pi_{B^{\prime}}: X^{B} \rightarrow X^{B^{\prime}}$ as $\boldsymbol{x}=\left(x_{1}, x_{2}, \ldots, x_{m}\right) \mapsto \pi_{B^{\prime}}(\boldsymbol{x})=\left(x_{1}, x_{2}, \ldots, x_{j}\right)$, and for a set of states $S^{B} \subseteq X^{B}$, we define $\pi_{B^{\prime}}\left(S^{B}\right)=\left\{\pi_{B^{\prime}}(\boldsymbol{x}): \boldsymbol{x} \in S^{B}\right\}$.

\subsection{Detect attractors in a block}

An elementary block does not depend on any other block while a non-elementary block does. Therefore, they can be treated separately. We first consider the case of elementary blocks. An elementary block is in fact a $\mathrm{BN}$; therefore, the definition of attractors in a BN can be directly taken to the concept of an elementary block.

Definition 7 (Preservation of attractors). Given a $B N G$ and an elementary block $B$ in $G$, let $\mathcal{A}=\left\{A_{1}, A_{2}, \ldots, A_{m}\right\}$ be the set of attractors of $G$ and $\mathcal{A}^{B}=\left\{A_{1}^{B}, A_{2}^{B}, \ldots\right.$, $\left.A_{m^{\prime}}^{B}\right\}$ be the set of attractors of $B$. We say that $B$ preserves the attractors of $G$ if for any $k \in[1, m]$, there is an attractor $A_{k^{\prime}}^{B} \in \mathcal{A}^{B}$ such that $\pi_{B}\left(A_{k}\right) \subseteq A_{k^{\prime}}^{B}$. 


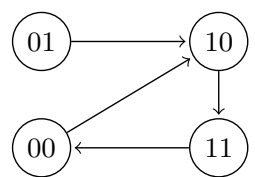

(a) Transition graph of Block $B_{1}$ in $G_{1}$.

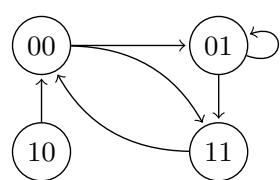

(b) Transition graph of the "realisation".

Fig. 3: Two transition graphs used in Example 3 and Example 4.

Example 3. Consider the $\mathrm{BN} G_{1}$ in Example 1. Its set of attractors is $\mathcal{A}=\{\{(000),(1 *$ $1)\}$ \}. Nodes $v_{1}$ and $v_{2}$ form an elementary block $B_{1}$. Since $B_{1}$ is an elementary block, it can be viewed as a BN. The transition graph of $B_{1}$ is shown in Figure 3a. Its set of attractors is $\mathcal{A}^{B_{1}}=\{\{(00),(1 *)\}\}$ (nodes are arranged as $\left.v_{1}, v_{2}\right)$. We have $\pi_{B_{1}}(\{(000),(1 *$ $1)\})=\{(00),(1 *)\} \in \mathcal{A}^{B_{1}}$, i.e., block $B_{1}$ preserves the attractors of $G_{1}$.

With Definition 7, we have the following lemma and theorem.

Lemma 1. Given a $B N G$ and an elementary block $B$ in $G$, let $\Phi$ be the set of attractor states of $G$ and $\Phi^{B}$ be the set of attractor states of $B$. If $B$ preserves the attractors of $G$, then $\Phi \subseteq \mathcal{M}_{G}\left(\Phi^{B}\right)$.

Theorem 1. Given a BN $G$, let $B$ be an elementary block in $G$. B preserves the attractors of $G$.

For an elementary block $B$, the mirror states of its attractor states cover all $G$ 's attractor states according to Lemma 1 and Theorem 1 . Therefore, by searching from the mirror states only instead of the whole state space, we can detect all the attractor states.

We now consider the case of non-elementary blocks.

Definition 8 (Parent SCC, Ancestor SCC). An SCC $\Sigma_{i}$ is called a parent SCC (or parent for short) of another SCC $\Sigma_{j}$ if $\Sigma_{i}$ contains at least one control node of $\Sigma_{j}$. Denote P $\left(\Sigma_{i}\right)$ the set of parent SCCs of $\Sigma_{i}$. An SCC $\Sigma_{k}$ is called an ancestor SCC (or ancestor for short) of an SCC $\Sigma_{j}$ if and only if either (1) $\Sigma_{k}$ is a parent of $\Sigma_{j}$ or (2) $\Sigma_{k}$ is a parent of $\Sigma_{k^{\prime}}$, where $\Sigma_{k^{\prime}}$ is an ancestor of $\Sigma_{j}$. Denote $\Omega\left(\Sigma_{j}\right)$ the set of ancestor SCCs of $\Sigma_{i}$.

For an SCC $\Sigma_{j}$, if it has no parent SCC, then this SCC can form an elementary block; if it has at least one parent, then it must have an ancestor that has no parent, and all its ancestors $\Omega\left(\Sigma_{j}\right)$ together can form an elementary block, which is also a BN. The SCC-based decomposition will usually result in one or more non-elementary blocks.

Definition 9 (Crossability, Cross operations). Let $G$ be a $B N$ and let $B_{i}$ be a nonelementary block in $G$ with the set of nodes $V^{B_{i}}=\left\{v_{p_{1}}, v_{p_{2}}, \ldots, v_{p_{s}}, v_{q_{1}}, v_{q_{2}}, \ldots\right.$, $\left.v_{q_{t}}\right\}$, where $q_{k}(k \in[1, t])$ are the indices of the control nodes also contained by $B_{i}$ 's parent block $B_{j}$ and $p_{k}(k \in[1, s])$ are the indices of the remaining nodes. We denote the set of nodes in $B_{j}$ as $V^{B_{j}}=\left\{v_{q_{1}}, v_{q_{2}}, \ldots, v_{q_{t}}, v_{r_{1}}, v_{r_{2}}, \ldots, v_{r_{u}}\right\}$, where $r_{k}(k \in[1, u])$ are the indices of the non-control nodes in $B_{j}$. Let further $\boldsymbol{x}^{B_{i}}=$ $\left(x_{1}, x_{2}, \ldots, x_{s}, y_{1}^{i}, y_{2}^{i}, \ldots, y_{t}^{i}\right)$ be a state of $B_{i}$ and $\boldsymbol{x}^{B_{j}}=\left(y_{1}^{j}, y_{2}^{j}, \ldots, y_{t}^{j}, z_{1}, z_{2}, \ldots\right.$, 
$\left.z_{u}\right)$ be a state of $B_{j}$. States $\boldsymbol{x}^{B_{i}}$ and $\boldsymbol{x}^{B_{j}}$ are said to be crossable, denoted as $\boldsymbol{x}^{B_{i}} \mathcal{C} \boldsymbol{x}^{B_{j}}$, if the values of their common nodes are the same, i.e., $y_{k}^{i}=y_{k}^{j}$ for all $k \in[1, t]$. The cross operation of two crossable states $\boldsymbol{x}^{B_{i}}$ and $\boldsymbol{x}^{B_{j}}$ is defined as $\Pi\left(\boldsymbol{x}^{B_{i}}, \boldsymbol{x}^{B_{j}}\right)=$ $\left(x_{1}, x_{2}, \ldots, x_{s}, y_{1}^{i}, y_{2}^{i}, \ldots, y_{t}^{i}, z_{1}, z_{2}, \ldots, z_{u}\right)$. The notion of crossability naturally extends to two blocks without common nodes: any two states of these blocks are crossable.

We say $S^{B_{i}} \subseteq X^{B_{i}}$ and $S^{B_{j}} \subseteq X^{B_{j}}$ are crossable, denoted as $S^{B_{i}} \mathcal{C} S^{B_{j}}$, if at least one of the sets is empty or the following two conditions hold: 1) for any state $\boldsymbol{x}^{B_{i}} \in S^{B_{i}}$, there exists a state $\boldsymbol{x}^{B_{j}} \in S^{B_{j}}$ such that $\boldsymbol{x}^{B_{i}}$ and $\boldsymbol{x}^{B_{j}}$ are crossable; 2) vice versa. The cross operation on two crossable non-empty sets of states $S^{B_{i}}$ and $S^{B_{j}}$ is defined as $\Pi\left(S^{B_{i}}, S^{B_{j}}\right)=\left\{\Pi\left(\boldsymbol{x}^{B_{i}}, \boldsymbol{x}^{B_{j}}\right) \mid \boldsymbol{x}^{B_{i}} \in S^{B_{i}}, \boldsymbol{x}^{B_{j}} \in S^{B_{j}}\right.$ and $\left.\boldsymbol{x}^{B_{i}} \mathcal{C} \boldsymbol{x}^{B_{j}}\right\}$. When one of the two sets is empty, the cross operation simply returns the other set, i.e., $\Pi\left(S^{B_{i}}, S^{B_{j}}\right)=S^{B_{i}}$ if $S^{B_{j}}=\emptyset$ and $\Pi\left(S^{B_{i}}, S^{B_{j}}\right)=S^{B_{j}}$ if $S^{B_{i}}=\emptyset$.

Let $\mathcal{S}^{B_{i}}=\left\{S^{B_{i}} \mid S^{B_{i}} \subseteq X^{B_{i}}\right\}$ be a family of sets of states in $B_{i}$ and $\mathcal{S}^{B_{j}}=$ $\left\{S^{B_{j}} \mid S^{B_{j}} \subseteq X^{B_{j}}\right\}$ be a family of sets of states in $B_{j}$. We say $\mathcal{S}^{B_{i}}$ and $\mathcal{S}^{B_{j}}$ are crossable, denoted as $\mathcal{S}^{B_{i}} \mathcal{C} \mathcal{S}^{B_{j}}$ if 1) for any set $S^{B_{i}} \in \mathcal{S}^{B_{i}}$, there exists a set $S^{B_{j}} \in$ $\mathcal{S}^{B_{j}}$ such that $S^{B_{i}}$ and $S^{B_{j}}$ are crossable; 2) vice versa. The cross operation on two crossable families of sets $\mathcal{S}^{B_{i}}$ and $\mathcal{S}^{B_{j}}$ is defined as $\Pi\left(\mathcal{S}^{B_{i}}, \mathcal{S}^{B_{j}}\right)=\left\{\Pi\left(S_{i}, S_{j}\right) \mid S_{i} \in\right.$ $\mathcal{S}^{B_{i}}, S_{j} \in \mathcal{S}^{B_{j}}$ and $\left.S_{i} \mathcal{C} S_{j}\right\}$.

Proposition 1. Let $V^{C}$ be the set of control nodes shared by two blocks $B_{i}$ and $B_{j}$, i.e., $V^{C}=V^{B_{i}} \cap V^{B_{j}}$ and let $S^{B_{i}} \subseteq X^{B_{i}}$ and $S^{B_{j}} \subseteq X^{B_{j}}$. Then $S^{B_{i}} \mathcal{C} S^{B_{j}}$ is equivalent to $\pi_{C}\left(S^{B_{i}}\right)=\pi_{C}\left(S^{B_{j}}\right)$.

After decomposing a BN into SCCs, there is at least one SCC with no control nodes. Hence, there is at least one elementary block in every BN. Moreover, for each nonelementary block we can construct, by merging all its predecessor blocks, a single parent elementary block. We detect the attractors of the elementary blocks and use the detected attractors to guide the values of the control nodes of their child blocks. The guidance is achieved by considering realisations of the dynamics of a non-elementary block with respect to the attractors of its parent elementary block, shortly referred to as realisations of a non-elementary block. In some cases, a realisation of a non-elementary block is simply obtained by assigning new Boolean functions to the control nodes of the block. However, in many cases, it is not this simple and a realisation of a non-elementary block is obtained by explicitly constructing a transition system of this block corresponding to the considered attractor of the elementary parent block. Since the parent block of a non-elementary block may have more than one attractor, a non-elementary block may have more than one realisation.

Definition 10 (Realisation of a non-elementary block). Let $B_{i}$ be a non-elementary block formed by merging an SCC with its control nodes. Let nodes $u_{1}, u_{2}, \ldots, u_{r}$ be all the control nodes of $B_{i}$ which are also contained in its elementary parent block $B_{j}$ (we can merge $B_{i}$ 's ancestor blocks to form $B_{j}$ if $B_{i}$ has more than one parent block or has a non-elementary parent block). Let $A_{1}^{B_{j}}, A_{2}^{B_{j}}, \ldots, A_{t}^{B_{j}}$ be the attractor systems of $B_{j}$. For any $k \in[1, t]$, a realisation of block $B_{i}$ with respect to $A_{k}^{B_{j}}$ is a state transition system such that

1. the transitions are as follows: for any transition $\boldsymbol{x}^{B_{j}} \rightarrow \tilde{\boldsymbol{x}}^{B_{j}}$ in the attractor system of $A_{k}^{B_{j}}$, there is a transition $\boldsymbol{x}^{B_{i, j}} \rightarrow \tilde{\boldsymbol{x}}^{B_{i, j}}$ in the realisation such that $\boldsymbol{x}^{B_{i, j}} \mathcal{C} \boldsymbol{x}^{B_{j}}$ 
and $\tilde{\boldsymbol{x}}^{B_{i, j}} \mathcal{C} \tilde{\boldsymbol{x}}^{B_{j}} ;$ each transition in the realisation is caused by the update of all nodes synchronously: the update of non-control nodes of $B_{i}$ is regulated by the Boolean functions of the nodes and the update of nodes in its parent block $B_{j}$ is regulated by the transitions of the attractor system of $A_{k}^{B_{j}}$;

In the realisation of a non-elementary block all the nodes of its single elementary parent block are considered and not only the control nodes of the parent block. This allows to distinguish the potentially different states in which the values of control nodes are the same. Without this, a state in the state transition graph of the realisation may have more than one out-going transition, which is contrary to the fact that the out-going transition for a state in a synchronous network is always determined. Although the definition of attractors can still be applied to such a transition graph, the attractor detection algorithms for synchronous networks, e.g., SAT-based algorithms, may not work any more. Moreover, the meaning of attractors in such a graph are not consistent with the synchronous semantics and therefore the detected "attractors" may not be attractors of the synchronous BN. Note that the decomposition method mentioned in [7] did not take care of this and therefore produces incorrect results in certain cases. We now give an example to illustrate one of such cases.

Example 4. Consider the BN in Example 1, which can be divided into two blocks: block $B_{1}$ with nodes $v_{1}, v_{2}$ and block $B_{2}$ with nodes $v_{2}, v_{3}$. The transition graph of $B_{1}$ is shown in Figure $3 \mathrm{a}$ and its attractor is $(00) \rightarrow(10) \rightarrow(11)$. If we do not include the node $v_{1}$ when forming the realisation of $B_{2}$, we will get a transition graph as shown in Figure $3 \mathrm{~b}$, which contains two states with two out-going transitions. This is contrary to the synchronous semantics. Moreover, recovering attractors with the attractors in this graph will lead to a non-attractor state of the original BN, i.e., (001).

For asynchronous networks, however, such a distinction is not necessary since the situation of multiple out-going transitions is in consistent with the asynchronous updating semantics. Definition 10 forms the basis for a key difference between this decomposition method for synchronous BNS and the one for asynchronous BNs proposed in [9].

Constructing realisations for a non-elementary block is a key process for obtaining its attractors. For each realisation, the construction process requires the knowledge of all the transitions in the corresponding attractor of its elementary parent block. In Section 4, we explain in details how to implement it with BDDs. We now give some examples.

Example 5. Consider the BN in Figure 2a. Its Boolean functions are given as follows:

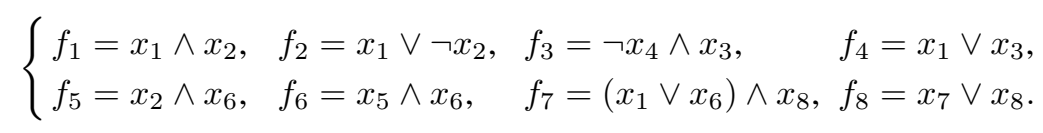

The network contains four SCCs $\Sigma_{1}, \Sigma_{2}, \Sigma_{3}$ and $\Sigma_{4}$. For any $\Sigma_{i}(i \in[1,4])$, we form a block $B_{i}$ by merging $\Sigma_{i}$ with its control nodes. Block $B_{1}$ is an elementary block and its transition graph is shown in Figure 2b. Block $B_{1}$ has two attractors, i.e., $\{(0 *)\}$ and $\{(11)\}$. Regarding the first attractor, block $B_{3}$ has a realisation by setting the nodes $v_{1}$ and $v_{2}$ (nodes from its parent block $B_{1}$ ) to contain transitions $\{(00) \rightarrow(01),(01) \rightarrow$ $(00)\}$. The transition graph of this realisation is shown in Figure 4a. Regarding the 


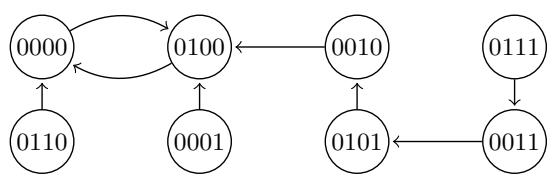

(a) Realisation 1 of Example 5.

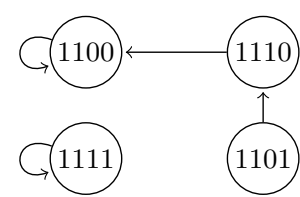

(b) Realisation 2 of Example 5.

Fig. 4: Transition graphs of two realisations in Example 5.

second attractor, block $B_{3}$ has a realisation by setting nodes $v_{1}$ and $v_{2}$ to contain only the transition $\{(11) \rightarrow(11)\}$. Its transition graph is shown in Figure $4 \mathrm{~b}$.

A realisation of a non-elementary block takes care of the dynamics of the undetermined nodes, providing a transition system of the block. Therefore, we can extend the attractor definition to realisations of non-elementary blocks as follows.

Definition 11 (Attractors of a non-elementary block). An attractor of a realisation of a non-elementary block is a set of states satisfying that any state in this set can be reached from any other state in this set and no state in this set can reach any other state that is not in this set. The attractors of a non-elementary block is the set of the attractors of all realisations of the block.

With the above definition, we can extend Definition 10 by allowing $B_{j}$ to be a nonelementary block as well. As long as $B_{i}$ 's parent block $B_{j}$ contains all the control nodes of block $B_{i}$, the attractors of $B_{j}$ can be used to form the realisations of $B_{i}$, no matter $B_{j}$ is elementary or not. Observe that using a non-elementary block as a parent block does not change the fact that the attractor states of the parent block contain the values of all the nodes in the current block and all its ancestor blocks.

Computing attractors for non-elementary blocks requires the knowledge of the attractors of its parent blocks. Therefore, we need to order the blocks so that for any block $B_{i}$, the attractors of its parent blocks are always detected before it. The blocks are ordered topologically. For easier explanation of the order, we introduce the concept of topological credit as follows. For simplification, we refer topological credit as credit in the remaining part of the paper.

Definition 12 (Topological credit). Given a $B N G$, the an elementary block $B_{i}$ of $G$ has a topological credit of 0 , denoted as $\mathcal{P}\left(B_{i}\right)=0$. Let $B_{j}$ be a non-elementary block and $B_{j_{1}}, \ldots, B_{j_{p(j)}}$ be all its parent blocks. The topological credit of $B_{j}$ is defined as $\mathcal{P}\left(B_{j}\right)=\max _{k=1}^{p(j)}\left(\mathcal{P}\left(B_{j_{k}}\right)\right)+1$, where $p(j)$ is the number of parent blocks of $B_{j}$.

\subsection{Recovering attractors for the original $\mathrm{BN}$}

After computing attractors for all the blocks, we need to recover attractors for the original $\mathrm{BN}$, with the help of the following theorem.

Theorem 2. Let $G$ be a BN and let $B_{i}$ be one of its blocks. Denote $\Omega\left(B_{i}\right)$ as the block formed by all $B_{i}$ 's ancestor blocks and denote $\mathcal{X}\left(B_{i}\right)$ as the block formed by merging $B_{i}$ with $\Omega\left(B_{i}\right) . \mathcal{X}\left(B_{i}\right)$ is in fact an elementary block, which is also a $B N$. The attractors of block $B_{i}$ are in fact the attractors of $\mathcal{X}\left(B_{i}\right)$. 
Theorem 3. Given a $B N G$, where $B_{i}$ and $B_{j}$ are its two blocks, let $\mathcal{A}^{B_{i}}$ and $\mathcal{A}^{B_{j}}$ be the set of attractors for $B_{i}$ and $B_{j}$, respectively. Let $B_{i, j}$ be the block got by merging the nodes in $B_{i}$ and $B_{j}$. Denote the set of all attractor states of $B_{i, j}$ as $S^{B_{i, j}}$. If $B_{i}$ and $B_{j}$ are both elementary blocks, $\mathcal{A}^{B_{i}} \mathcal{C} \mathcal{A}^{B_{j}}$ and $\cup_{A \in \Pi\left(\mathcal{A}^{\left.B_{i}, \mathcal{A}^{B_{j}}\right)}\right.} A=S^{B_{i, j}}$.

The above developed theoretical background with Theorem 2 and Theorem 3 being its core result, allows us to design a new decomposition-based approach towards detection of attractors in large synchronous BNs. The idea is as follows. We divide a BN into blocks according to the detected SCCs. We order the blocks in the ascending order based on their credits and detect attractors of the ordered blocks one by one in an iterative way. We start from detecting attractors of elementary blocks (credit 0 ), and continue to detect blocks with higher credits after constructing their realisations. According to Theorem 2, by detecting the attractors of a block, we in fact obtain the attractors of the block formed by the current block and all its ancestor blocks. Hence, after the attractors of all the blocks have been detected, either we have obtained the attractors of the original BN or we have obtained the attractors of several elementary blocks of this BN. According to Theorem 3, we can perform a cross operation for any two elementary blocks (credits $0)$ to recover the attractor states of the two merged blocks. The resulting merged block will form a new elementary block, i.e., one with credit 0 . The attractors can be easily identified from the set of attractor states. By iteratively performing the cross operation until a single elementary block containing all the nodes of the $\mathrm{BN}$ is obtained, we can recover the attractors of the original $\mathrm{BN}$. The details of this new algorithm are discussed in the next section. In addition, we have the following corollary that extends Theorem 3 by allowing $B_{i}$ and $B_{j}$ to be non-elementary blocks. This corollary will be used in the next section.

Corollary 1. Given a $B N G$, where $B_{i}$ and $B_{j}$ are its two blocks, let $\mathcal{A}^{B_{i}}$ and $\mathcal{A}^{B_{j}}$ be the set of attractors for $B_{i}$ and $B_{j}$, respectively. Let $B_{i, j}$ be the block got by merging the nodes in $B_{i}$ and $B_{j}$. Denote the set of attractor states of $B_{i, j}$ as $S^{B_{i, j}}$. It holds that $\mathcal{A}^{B_{i}} \mathcal{C} \mathcal{A}^{B_{j}}$ and $\cup_{S \in \Pi\left(\mathcal{A}^{B_{i}}, \mathcal{A}^{B_{j}}\right)} S=S^{B_{i, j}}$.

\section{A BDD-based Implementation}

We describe the SCC-based attractor detection method in Algorithm 1. As mentioned in Section 2.2, we encode BNs in BDDs; hence most operations in this algorithm is performed with BDDs. Algorithm 1 takes a $\mathrm{BN} G$ and its corresponding transition system $\mathcal{T}$ as inputs, and outputs the set of attractors of $G$. In this algorithm, we denote by $\operatorname{DETECT}(\mathcal{T})$ a basic function for detecting attractors of a given transition system $\mathcal{T}$. Lines 23-26 of this algorithm describe the process for detecting attractors of a nonelementary block. The algorithm detects the attractors of all the realisations of the nonelementary block and performs the union operation of the detected attractors. For this, if the non-elementary block has only one parent block, its attractors are already computed as the blocks are considered in the ascending order with respect to their credits by the main for loop in line 4. Otherwise, all the parent blocks are considered in the for loop in lines 13-21. By iteratively applying the cross operation in line 16 to the attractor sets of the ancestor blocks in the ascending order, the attractor states of a new block formed 


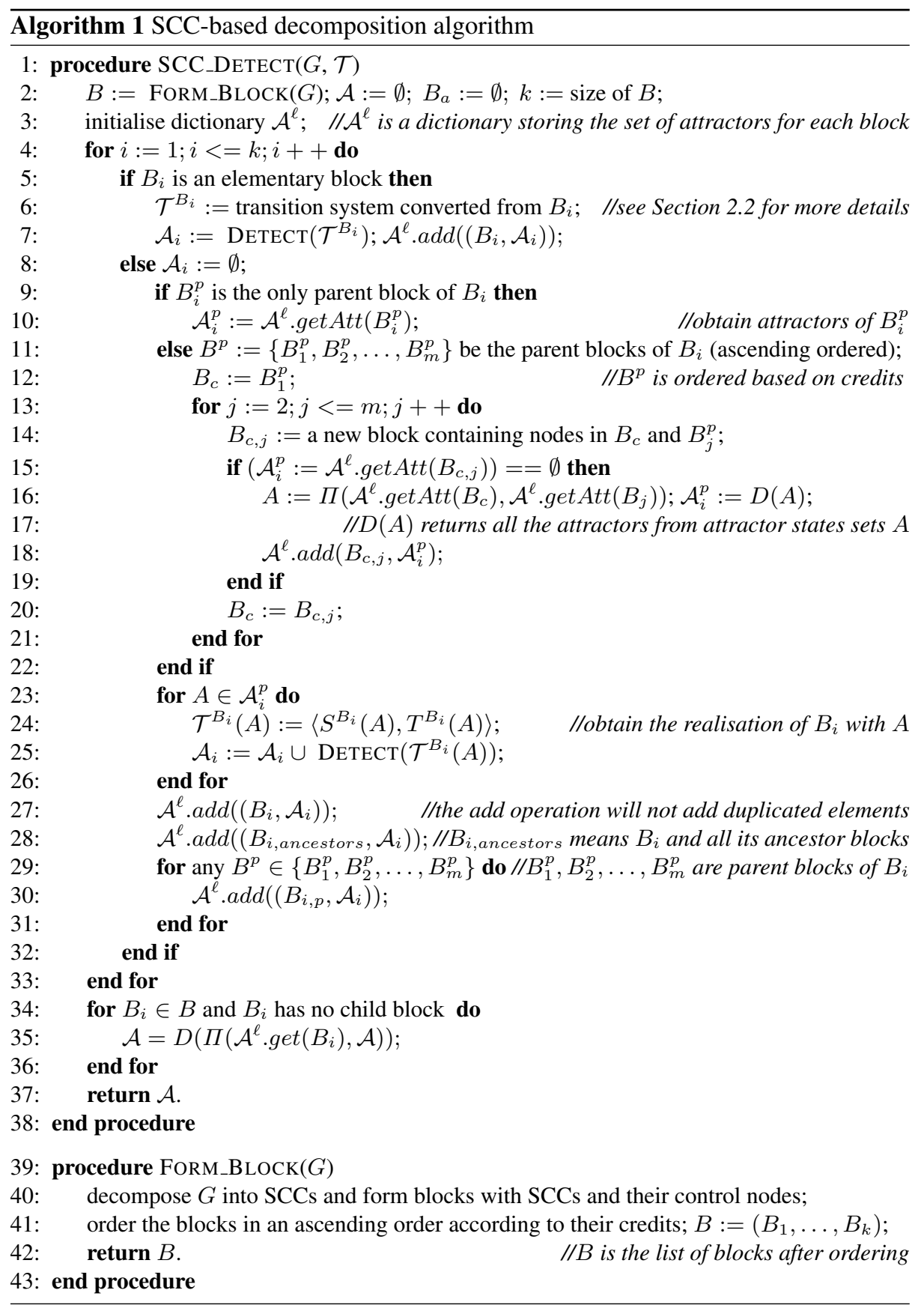

by merging all the parent blocks are computed as assured by Corollary 1 . The attractors 


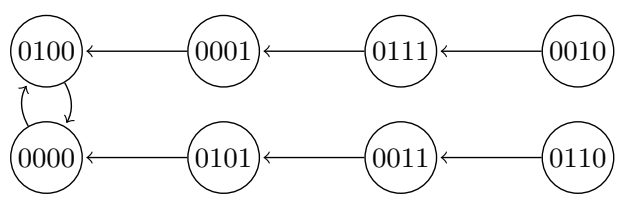

(a) Realisation 1 of $B_{2}$.

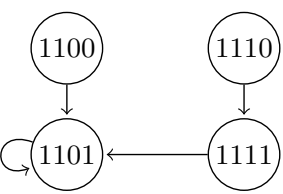

(b) Realisation 2 of $B_{2}$.

Fig. 5: Two realisations used in Example 6.

are then identified from the attractor states with one more operation. The correctness of the algorithm is stated as Theorem 4.

Theorem 4. Algorithm 1 correctly identifies the set of attractors of a given BN G.
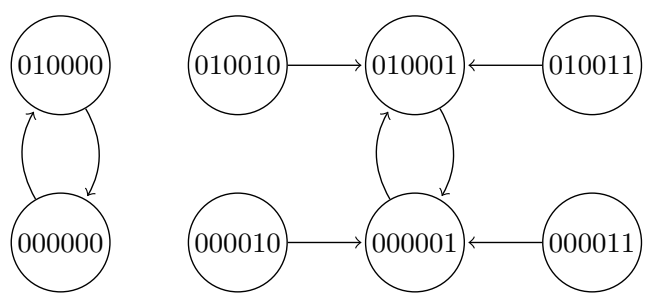

(a) The first realisation of $B_{4}$

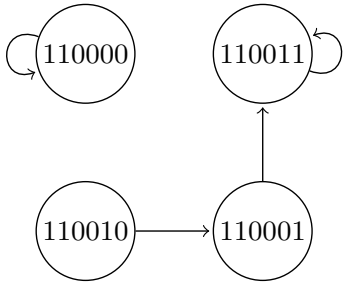

(b) The second realisation of $B_{4}$

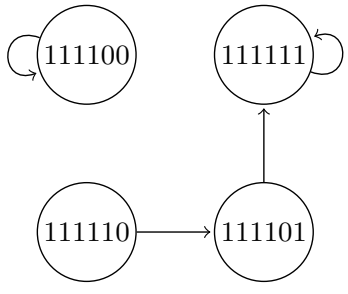

(c) The third realisation of $B_{4}$

Fig. 6: Transition graphs of the three realisations for block $B_{4}$.

We continue to illustrate in Example 6 how Algorithm 1 detects attractors.

Example 6. Consider the BN shown in Example 5 and its four blocks. Block $B_{1}$ is an elementary block and it has two attractors, i.e., $\mathcal{A}_{1}=\{\{(0 *)\},\{(11)\}\}$. To detect the attractors of block $B_{2}$, we first form realisations of $B_{2}$ with the attractors of its parent block $B_{1}$. $B_{1}$ has two attractors so there are two realisations for $B_{2}$. The transition graphs of the two realisations are shown in Figures 5a and 5b. We get two attractors for block $B_{2}$, i.e., $\mathcal{A}_{2}=\{\{(0 * 00)\},\{(1101)\}\}$. Those two attractors are also attractors for the merged block $B_{1,2}$, i.e., $\mathcal{A}_{1,2}=\mathcal{A}_{2}$. In Example 5, we have shown the two realisations of $B_{3}$ regarding the two attractors of $B_{1}$. Clearly, $B_{3}$ has two attractors, i.e., $\mathcal{A}_{3}=\{\{(0 * 00)\},\{(1100)\},\{(1111)\}\} . B_{4}$ has two parent blocks. Therefore, we need 


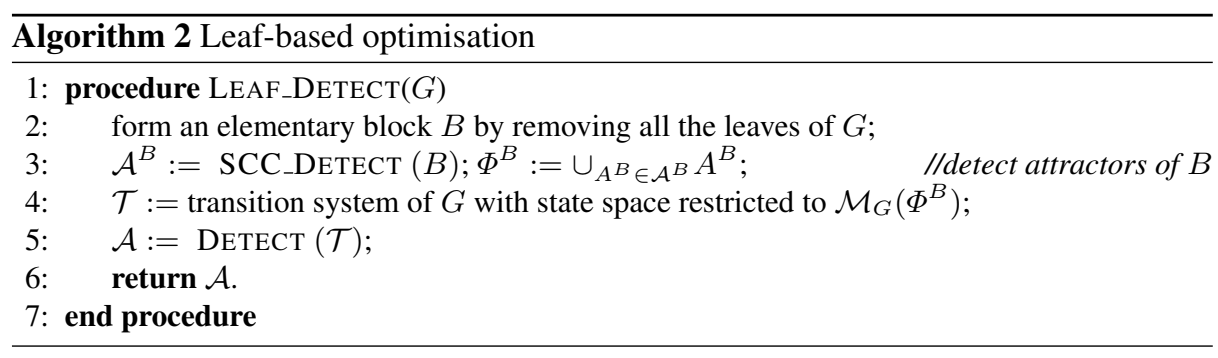

to merge the two parent blocks to form a single parent block. Since the attractors of the merged block $B_{1,3}$ are the same as $B_{3}$, we directly obtain the attractors of $B_{1,3}$, i.e., $\mathcal{A}_{1,3}=\mathcal{A}_{3}=\{\{(0 * 00)\},\{(1100),(1111)\}\}$. There are three attractors so there will be three realisations for block $B_{4}$. The transition graph of the three realisations are shown in Figure 6. From the transition graphs, we easily get the attractors of $B_{4}$, i.e., $\mathcal{A}_{4}=$ $\{\{(0 * 0000)\},\{(0 * 0001)\},\{(110000)\},\{(110011)\},\{(111100)\},\{(111111)\}\}$. Now the attractors for all the blocks have been detected. We can now obtain all the attractors of the $\mathrm{BN}$ by several cross operations. We start from the block with the largest credit, i.e., block $B_{4}$. The attractors of $B_{4}$ in fact cover blocks $B_{1}, B_{3}$ and $B_{4}$. The remaining block is $B_{2}$. We perform a cross operation on $\mathcal{A}_{2}$ and $\mathcal{A}_{4}$ and based on the obtained result we detect the attractors of the BN, i.e., $\mathcal{A}=D\left(\Pi\left(\mathcal{A}_{2}, \mathcal{A}_{4}\right)=\{\{(0 *\right.$ $000000)\},\{(0 * 000001)\},\{(11010011)\},\{(11010000)\},\{(11011111)\},\{(11011100)\}\}$.

\subsection{An optimisation}

It often happens that a BN contains many leaf nodes that do not have any child node. Each of the leaf nodes will be treated as an SCC in our algorithm and it is not worth the effort to process an SCC with only one leaf node. Therefore, we treat leaf nodes in a special way. Formally, leaf nodes are recursively defined as follows.

Definition 13. A node in a BN is a leaf node (or leaf for short) if and only if it is not the only node in the BN and either (1) it has no child nodes except for itself or (2) it has no other children after iteratively removing all its child nodes which are leaf nodes.

Algorithm 2 outlines the leaf-based decomposition approach for attractor detection. We now show that Algorithm 2 can identify all attractor states of a given BN.

Theorem 5. Algorithm 2 correctly identifies all the attractor states of a given BN G.

\section{Experimental Results}

We have implemented the decomposition algorithm presented in Section 4 in the model checker MCMAS [14]. In this section, we demonstrate the efficiency of our method by comparing our method with the state-of-the-art decomposition method mentioned in [8] which is also based on BDD implementation. We generate 33 random BN models with different number of nodes using the tool ASSA-PBN $[15,16]$ and compare the 


\begin{tabular}{rccc|rrr|rrr}
\hline model & $\begin{array}{c}\# \\
\text { ID non- }\end{array}$ & nodes leaves attractors & \multicolumn{3}{|c|}{ original models } & \multicolumn{3}{|c}{ models with leaves removed } \\
$t_{M_{2}}[s]$ & $t_{M_{1}}[s]$ & Speedup & $t_{M_{2}}[s]$ & $t_{M_{1}}[s]$ & Speedup \\
\hline 1 & 100 & 7 & 32 & 4.56 & 0.86 & 5.3 & 0.58 & 0.02 & 29.0 \\
2 & 120 & 9 & 1 & 18.13 & 0.95 & 19.1 & 1.10 & 0.04 & 27.5 \\
3 & 150 & 19 & 2 & 201.22 & 1.66 & 121.2 & 0.74 & 0.02 & 37.0 \\
4 & 200 & 6 & 16 & 268.69 & 7.04 & 38.2 & 0.97 & 0.02 & 48.5 \\
5 & 250 & 25 & 12 & 533.57 & 11.16 & 47.8 & 0.90 & 0.04 & 22.5 \\
6 & 300 & 88 & 1 & - & - & N/A & 238.96 & 65.33 & 3.7 \\
7 & 450 & 43 & 8 & - & 60.82 & N/A & 3704.33 & 0.17 & 21790.2 \\
\hline
\end{tabular}

Table 1: Selected results for the performance comparison of methods $M_{1}$ and $M_{2}$.

performance of the two methods on these 33 models. All the experiments are conducted on a computer with an Intel Xeon W3520@2.67GHz CPU and 12GB memory.

We name our proposed decomposition method $M_{1}$ and the one in [8] $M_{2}$. There are two possible implementations of the DETECT function used in Algorithm 1 as mentioned in [8]: monolithic and enumerative. We use the monolithic one which is shown to be more suitable for small networks as the decomposed sub-networks are relatively small. Since the method in [8] uses similar leaf reduction technique, we make comparisons on both the original models and the models whose leaves are removed in order to eliminate the influence of leaf nodes. We set the expiration time to 3 hours. Before removing leaf nodes, there are 11 cases that both methods fail to process. Among the other 22 cases, our method is faster than $M_{2}$ in 16 cases, which is approximately $73 \%$. After removing leaf nodes, there are 5 cases that both methods fail to process. Among the other 28 cases, our method is faster than $M_{2}$ in 25 cases, which is approximately $89 \%$. We demonstrate the results for 7 models in Table 1 and the remaining result can be found in [17]. Since our method considers the dependency relation between different blocks, the attractors of all the blocks need to be computed; while method $M_{2}$ can ignore the blocks with only leaf nodes. Therefore, the performance of our method is more affected by the leaf nodes. This is why the percentage that our method is faster than $M_{2}$ is increased from $73 \%$ to $89 \%$ when leaf nodes are removed. Notably, after eliminating the influence of leaf nodes, our method is significantly faster than $M_{2}$. The "-" in Table 1 means the method fails to process the model within 3 hours. The speedup is therefore not applicable (N/A) for this result. The speedup is computed as $t_{M_{2}} / t_{M_{1}}$, where $t_{M_{1}}$ is the time cost for $M_{1}$ and $t_{M_{2}}$ is the time cost for $M_{2}$. All the time shown in Table 1 is in seconds. In general, we obtain a larger speedup when the number of attractors is relatively small. This is due to that our method takes the attractors of the parent block into account when forming a realisation of a non-elementary block and the number of realisations increases with the number of attractors. Summarising, our new method shows a significant improvement on the state-of-the-art decomposition method.

\section{Conclusion and Future Work}

We have introduced a new SCC-based decomposition method for attractor detection of large synchronous BNs. Although our decomposition method shares similar ideas on how to decompose a large network with existing decomposition methods, our method differs from them in the key process and has significant advantages. 
First, our method is designed for synchronous BNs, as a consequence the key process for constructing realisations in our method is totally different from the one in [9], which is designed for asynchronous networks. Secondly, our method considers the dependency relation among the sub-networks. The method in [8] does not rely on this relation and only takes the detected attractors in sub-networks to restrict the initial states when recovering the attractors for the original network. In this way, the decomposition method in [8] potentially cannot scale up very well for large networks, as it still requires a BDD encoding of the transition relation of the whole network. This is our main motivation to extend our previous work [9] towards synchronous BNs. Experimental results show that our method is significantly faster than the one in [8]. Lastly, we have shown that the method proposed in [7] cannot compute correct results in certain cases.

Our current implementation is based on BDDs. One future work is to use SATsolvers to implement the DETECT function as SAT-based methods are normally more efficient in terms of attractor detection for synchronous BNs [6].

\section{References}

1. Kauffman, S.: Homeostasis and differentiation in random genetic control networks. Nature 224 (1969) 177-178

2. Huang, S.: Genomics, complexity and drug discovery: insights from Boolean network models of cellular regulation. Pharmacogenomics 2(3) (2001) 203-222

3. Somogyi, R., Greller, L.D.: The dynamics of molecular networks: applications to therapeutic discovery. Drug Discovery Today 6(24) (2001) 1267-1277

4. Garg, A., Xenarios, L., Mendoza, L., DeMicheli, G.: An efficient method for dynamic analysis of gene regulatory networks and in silico gene perturbation experiments. In: Proc. 11th Annual Conference on Research in Computational Molecular Biology. Volume 4453 of LNCS., Springer (2007) 62-76

5. Garg, A., Di Cara, A., Xenarios, I., Mendoza, L., De Micheli, G.: Synchronous versus asynchronous modeling of gene regulatory networks. Bioinformatics 24(17) (2008) 19171925

6. Dubrova, E., Teslenko, M.: A SAT-based algorithm for finding attractors in synchronous Boolean networks. IEEE/ACM Transactions on Computational Biology and Bioinformatics 8(5) (2011) 1393-1399

7. Guo, W., Yang, G., Wu, W., He, L., Sun, M.: A parallel attractor finding algorithm based on Boolean satisfiability for genetic regulatory networks. PLOS ONE 9(4) (2014) e94258

8. Yuan, Q., Qu, H., , Pang, J., Mizera, A.: Improving BDD-based attractor detection for synchronous Boolean networks. Science China Information Sciences 59(8) (2016) 080101

9. Mizera, A., Pang, J., Qu, H., Yuan, Q.: Taming asynchrony for attractor detection in large Boolean networks (technical report). Available online at http://arxiv.org/abs/ 1704.06530 (2017)

10. Kauffman, S.A.: Metabolic stability and epigenesis in randomly constructed genetic nets. Journal of Theoretical Biology 22(3) (1969) 437-467

11. Shmulevich, I., Dougherty, E.R.: Probabilistic Boolean Networks: The Modeling and Control of Gene Regulatory Networks. SIAM Press (2010)

12. Lee, C.Y.: Representation of switching circuits by binary-decision programs. Bell System Technical Journal 38(4) (1959) 985-999

13. Akers, S.B.: Binary decision diagrams. IEEE Transactions on Computers 100(6) (1978) 509-516 
14. Lomuscio, A., Qu, H., Raimondi, F.: MCMAS: An open-source model checker for the verification of multi-agent systems. International Journal on Software Tools for Technology Transfer (2015)

15. Mizera, A., Pang, J., Yuan, Q.: ASSA-PBN: a tool for approximate steady-state analysis of large probabilistic Boolean networks. In: Proc. 13th International Symposium on Automated Technology for Verification and Analysis. Volume 9364 of LNCS., Springer (2015) 214-220

16. Mizera, A., Pang, J., Yuan, Q.: ASSA-PBN 2.0: A software tool for probabilistic Boolean networks. In: Proc. 14th International Conference on Computational Methods in Systems Biology. Volume 9859 of LNCS., Springer (2016) 309-315

17. Mizera, A., Pang, J., Qu, H., Yuan, Q.: Benchmark Boolean networks. http://satoss . uni.lu/software/ASSA-PBN/benchmark/attractor_syn.xlsx 